\title{
High-precision volume-staged Gamma Knife surgery and equivalent hypofractionation dose schedules for treating large arteriovenous malformations
}

\author{
Clinical article
}

\author{
Shannon Fogh, M.D., ${ }^{1}$ Lijun Ma, Ph.D., ${ }^{1}$ Nalin Gupta, Ph.D., ${ }^{2}$ Arjun Sahgal, M.D., ${ }^{3}$ \\ Jean L. Nakamura, M.D., ${ }^{1}$ IgOR Barani, M.D., ${ }^{1}$ Penny K. Sneed, M.D., ${ }^{1}$ \\ Michael McDermott, M.D., ${ }^{2}$ ANd David A. Larson, M.D., PH.D. ${ }^{1}$
}

Departments of ${ }^{1}$ Radiation Oncology and ${ }^{2}$ Neurosurgery, University of California, San Francisco, California; and ${ }^{3}$ Department of Radiation Oncology, Sunnybrook Health Sciences Centre, Princess Margaret Hospital, University of Toronto, Ontario, Canada

\begin{abstract}
Object. The goal of this study was to develop a technique for performing submillimeter high-precision volumestaged Gamma Knife surgery and investigate its potential benefits in comparison with hypofractionated stereotactic radiotherapy (SRT) for treating large arteriovenous malformations (AVMs).

Methods. The authors analyzed 7 pediatric AVM cases treated with volume-staged stereotactic radiosurgery (SRS) using the Gamma Knife Perfexion at the University of California, San Francisco. The target and normal tissue contours from each case were exported for hypofractionated treatment planning based on the Gamma Knife Extend system or the CyberKnife SRT. Both the Gamma Knife Extend and CyberKnife treatment plans were matched to yield the same level of target coverage (95\%-98\%) and conformity indices (1.24-1.46). Finally, hypofractionated treatment plans were compared with volume-staged treatment plans for sparing normal brain by using biologically equivalent 12-Gy normal brain volumes.

Results. Hypofractionated Gamma Knife Extend and CyberKnife treatment plans exhibited practically identical sparing of normal brain for the studied cases. However, when matching such values with volume-staged treatments for the biological effective dose, only conservative dose fractionation schemes, such as $27.3 \mathrm{~Gy}$ in 5 fractions and 25 Gy in 4 fractions, were found to be comparable to the volume-staged treatments. On average, this represents a mean $18.7 \% \pm 7.3 \%$ reduction in the single-fraction biologically equivalent dose for hypofractionated treatments versus the reference volume-staged treatments $(\mathrm{p}<0.001)$.

Conclusions. Volume staging remains advantageous over hypofractionation in delivering a higher dose to the target and for better sparing of normal brain tissue in the treatment of large AVMs. More clinical data are needed, however, to justify the clinical superiority of this increased dose when compared with a hypofractionated treatment regimen.
\end{abstract}

(http://thejns.org/doi/abs/10.3171/2012.7.GKS121023)

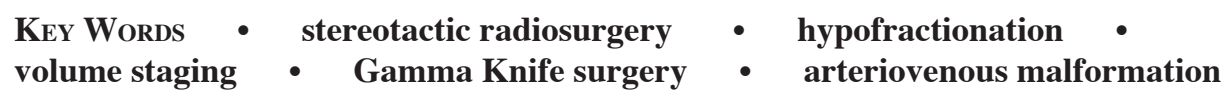

$\mathrm{S}$ TEREOTACTIC radiosurgery is a valuable therapeutic modality in the management of large $\mathrm{AVMs}^{5,8,10,12 \text {, }}$ 13,23 and has been adopted as an effective way to deliver a high dose of radiation in a highly conformal manner to maximally spare surrounding normal brain tissue and minimize the risk of radiation injury. ${ }^{8,13,21}$ Traditionally, the strategy for safely treating large AVMs at the University of California, San Francisco, has been a volume-staged SRS approach. This approach is used when the volume of the target is too large to safely deliver a single-fraction dose high enough to obliterate the target while maintaining the surrounding area treated by 12 Gy at an acceptable threshold. This is because the risk

Abbreviations used in this paper: $\mathrm{AVM}=$ arteriovenous malformation; GKS = Gamma Knife surgery; LINAC = linear accelerator; $\mathrm{SRS}=$ stereotactic radiosurgery; SRT = stereotactic radiotherapy. of post-SRS brain necrosis specific to AVM treatment has been found to correlate with the volume of normal brain surrounding the irradiated target area, which is exposed to $12 \mathrm{~Gy} .3,6,7,18,19$ The technique involves first treating a portion of the target volume (typically the area of the nidus close to feeder veins) with the intended SRS dose, followed by treatments of remaining portions of the target volume in a sequential manner.

With the recent introduction of precision SRT technologies, such as the Gamma Knife Extend system, and LINAC-based modalities, such as the CyberKnife, hypofractionated SRT, in which a high dose is delivered to the target volume in 5 or fewer fractions, has been proposed..$_{15,16,24,25}$ In particular, physician experiences with CyberKnife $^{1}$ (Accuray) treatments that have been reported in the literature indicate encouraging preliminary results. ${ }^{5,20,23}$ 
While a potential advantage to using hypofractionation is the reproducibility of targeting the same area without the risk of dynamic changes that may occur between treatment delivery sessions in a multistaged treatment, a key challenge is how to deliver a sufficiently high total dose to produce efficacy comparable to that of single-fraction SRS. ${ }^{9}$ In addition, while hypofractionated treatments theoretically allow better sparing of normal brain tissue and target dose delivery compared with volume-staged treatments, it is difficult to quantify a comparison between a traditional hypofractionated regimen and a volume-staged regimen.

The goal of our study was twofold: 1) to develop a volume-staged technique with accuracy comparable to that of existing SRT delivery; and 2) to develop data-driven hypofractionated treatment protocols by comparing biologically equivalent normal brain 12-Gy volumes from a hypofractionated treatment with those of a volume-staged Gamma Knife treatment.

\section{Methods}

Seven children with AVMs who had undergone volume-staged treatment with the Gamma Knife Perfexion (Elekta AB) at the University of California, San Francisco, were selected for the study. The mean target volume for these cases was $11.3 \pm 2.2 \mathrm{~cm}^{3}$ (range $8.0-15.0 \mathrm{~cm}^{3}$ ). All patients underwent 2 volume-staged GKSs. The technical details of our volume-staging technique have been previously described..$^{18}$ In brief, AVM target volumes are divided into 2 approximately equal portions, with each portion treated to a dose of $17 \mathrm{~Gy}$ or higher; the time interval between the 2 treatments is typically $6-9$ months. To match the 2 volume-staged treatment plans, we developed a 3-dimensional image registration plus a landmarkbased frame coordinate transformation procedure. The key goal of the procedure was to translate the Gamma Knife shot or isocenter coordinates from 1 frame system to another. Our procedure involved the following steps: 1) at both sessions, define the time-of-flight MR target image according to the treatment-day frame system; 2) at the second treatment session, coregister the MRI study obtained that day with the initial-frame MRI study obtained on the first treatment day via a voxel-based mutual information matching method; 3 ) identify the same landmark coordinates on the 2 imaging studies described in the first 2 steps; and 4) translate the radiation shot positions from the earlier treatment based on identified landmarks, which intrinsically specifies the 2 frame systems. Implementation of the fourth step is similar to that described in a previous study, ${ }^{18}$ except that the shot transformation matrix is determined via a nonlinear gradient search algorithm in an Excel spreadsheet with an embedded solver package (the spreadsheet is available and free from the corresponding author upon request).

For each volume-staged case, a corresponding Gamma Knife Extend plan was reconstructed. In addition, the imaging studies plus associated contour lines were exported via the DICOM-RT protocol using the Leksell GammaPlan image export tool (version 9.0, Elekta AB). The exported images and contour lines were then imported into the CyberKnife MultiPlan workstation (version
3.5, Accuray) for independent treatment planning. Since both Gamma Knife Extend system and CyberKnife treatments require a CT study for dose computation, a reference phantom CT study was used to merge with the MRI studies obtained in the pediatric patients for treatment plan optimization in a consistent manner. The CyberKnife treatment plans were optimized via the sequential inverse optimization routine. For all treatment plans, dose-volume constraints were imposed to produce a comparable dose to surrounding normal brain tissue while achieving the same target volume coverage as the corresponding Gamma Knife Extend system treatment plans.

Once a hypofractionated treatment plan had been developed, the hypofractionated total dose for each case was determined to produce an identical volume of normal brain receiving $12 \mathrm{~Gy}$-the $12-\mathrm{Gy}$ isodose volume minus the target volume, in biological equivalence to the corresponding volume-staged Gamma Knife treatment plan. For the purpose of illustration, suppose that a composite volume receiving 12 Gy in a volume-staged Gamma Knife treatment plan was found to equal $15.0 \mathrm{~cm}^{3}$, then when setting the target dose for an n-fraction SRT treatment, we would first adjust the prescribed dose value such that the plan produced exactly $15 \mathrm{~cm}^{3}$ of $12 \mathrm{~Gy}$ according to the biologically effective dose. In the context of the standard linear quadratic model, this means that $15 \mathrm{~cm}^{3}$ of the biologically effective dose equals $60 \mathrm{~Gy}_{3}$, that is, 12 $\times(1+12 / 3)=60 \mathrm{~Gy}_{3}$ where $\alpha / \beta=3 \mathrm{~Gy}$ is taken for the normal brain. ${ }^{11}$ Converting this into a hypofractionated n-fraction treatment, we can further compute a normal brain dose of $d_{T}$ of any given $n$-fraction scheme as follows: $d_{T}\left(1+d_{T} / 3 \mathrm{n}\right)=60$ or $d_{T}=3 / 2\left[\operatorname{Sqrt}\left(80 n+n^{2}\right)-n\right]$.

Therefore, for an $n$-fraction SRT treatment, this means that the target dose can be adjusted so that the isodose volume of $d_{T}$ would match the known volume receiving 12 Gy from the staged treatment (such as $15 \mathrm{~cm}^{3}$ in the example given previously). From Equation 1, we have $d_{T}=23.4$ Gy if $\mathrm{n}=5, \mathrm{~d}_{T}=21.5$ Gy if $\mathrm{n}=4, \mathrm{~d}_{T}=19.2 \mathrm{~Gy}$ if $\mathrm{n}=3$, and so forth.

Furthermore, the prescribed dose can be also be estimated based on an analytical model from our previous study, ${ }^{17}$ in which the prescribed target dose $\left(\mathrm{d}_{0}\right)$ relating to a nearby peripheral isodose volume for a solitary target is given as follows: $V_{0} / V_{T}=\left(d_{0} / d_{T}\right)^{\gamma}$, where $V_{0}$ is the prescribed isodose volume, $V_{T}$ is the isodose volume corresponding to $d_{T}$, and $\gamma$ is the power index approximately equal to -1.5 for radiosurgical deliveries.

Using the aforementioned example, suppose a target of $V_{0}=11 \mathrm{~cm}^{3}$ and a volume receiving $12 \mathrm{~Gy}$ of $V_{T}=15.0$ $\mathrm{cm}^{3}$. Substituting $d_{T}=23.4$ Gy for a 5 -fraction treatment of Equation 1 results in Equation 2, one can then obtain an estimated target prescribed dose of $d_{0}=28.8 \mathrm{~Gy}$ in 5 fractions. This value was taken as the initial solution for individual treatment plans. For example, once such an estimated value is set for a case, the corresponding $V_{T}$ can be obtained. Depending on the differences between the computed $V_{T}$ and the desired $V_{T}$ value, the target dose can be then iteratively adjusted such that the resulting $V_{T}$ of the $\mathrm{n}$-fraction treatment plan matches that determined from Equation 1. 


\section{Results}

Our overall accuracy in the volume-stage frame matching for all the cases was a mean of $0.33 \pm 0.09 \mathrm{~mm}$ with the maximum less than $0.5 \mathrm{~mm}$. Such a result indicated that the whole-procedure accuracy of a volumestaged SRT agrees with the accuracy of the standard GKS to within $0.5 \mathrm{~mm}$, a value approaching the mechanical accuracy of the latest Gamma Knife Perfexion system. ${ }^{14}$

The isodose distributions of a volume-staged Gamma Knife-treated AVM case are shown in Fig. 1A and B. The corresponding hypofractionated treatment plans are shown in Fig. 1C (CyberKnife) and Fig. 1D (Gamma Knife Extend system). The composite 12-Gy isodose volume (the isodose volume receiving 12 Gy, which is arrived at by summing the dose distributions of Fig. 1A and $\mathrm{B}$ ) was $21.3 \mathrm{~cm}^{3}$ for the case. When matching the biologically effective 12-Gy volume for either SRT plan, a prescription dose of 29 Gy in 5 fractions was derived for the case.

The treatment plan summary for all case results is shown in Table 1. Because of the conformity and target coverage constraints imposed on the CyberKnife SRT and Gamma Knife Extend SRT plans for this study, the resulting 12-Gy isodose volumes agreed within 0.2 $\mathrm{cm}^{3}$ between the 2 systems and these values are shown in Table 1. For all cases, a reduction of $18.7 \% \pm 7.3 \%$ in the equivalent single fractional dose was observed for the SRT plans to maintain the same 12-Gy isodose volumes as those of the volume-staged SRS treatments. Such a difference was statistically significant $(p=0.0014)$ based on the paired 2-tail Student t-test. The mean composite 12Gy isodose volume for the staged treatments was $22.6 \pm$ $5.6 \mathrm{~cm}^{3}$ versus $28.6 \pm 6.9 \mathrm{~cm}^{3}$ for the SRTs.

The mean 12-Gy isodose volume to the ventricles adjacent the targets was $0.35 \pm 0.48 \mathrm{~cm}^{3}$ for the staged treatments versus $0.63 \pm 1.04 \mathrm{~cm}^{3}$ for the SRTs $(p=0.245)$. Subtracting the enclosed portion of the ventricle volumes and the target volumes from the total 12-Gy isodose volumes, as listed in Table 1, we obtained mean normalbrain 12-Gy volumes of $22.2 \pm 5.5 \mathrm{~cm}^{3}$ for the staged treatments and $27.9 \pm 6.7 \mathrm{~cm}^{3}$ for the hypofractionated SRTs $(\mathrm{p}=0.002)$.

The prescribed radiation dose guideline for SRTs, as derived from the studied cases, is shown in Fig. 2. The error bars on the plot represent 1 standard deviation value. From the results in Fig. 2, dose variations among individual cases tend to increase nonlinearly with increasing number of fractions. Based on the fitted curve, the most common 5-fractionation dose scheme for an SRT would be $27.3 \mathrm{~Gy}$.

\section{Discussion}

We determined hypofractionation treatment schemes for large AVMs that would yield equivalent sparing of normal brain tissue to that of volume-staged GKS. The doses that our model derived are fundamentally based on the biologically equivalent 12 -Gy single-fraction isodose volume as the predictor of treatment-related toxicity. The total doses plotted in Fig. 2 (for example, 27.0 Gy in 5

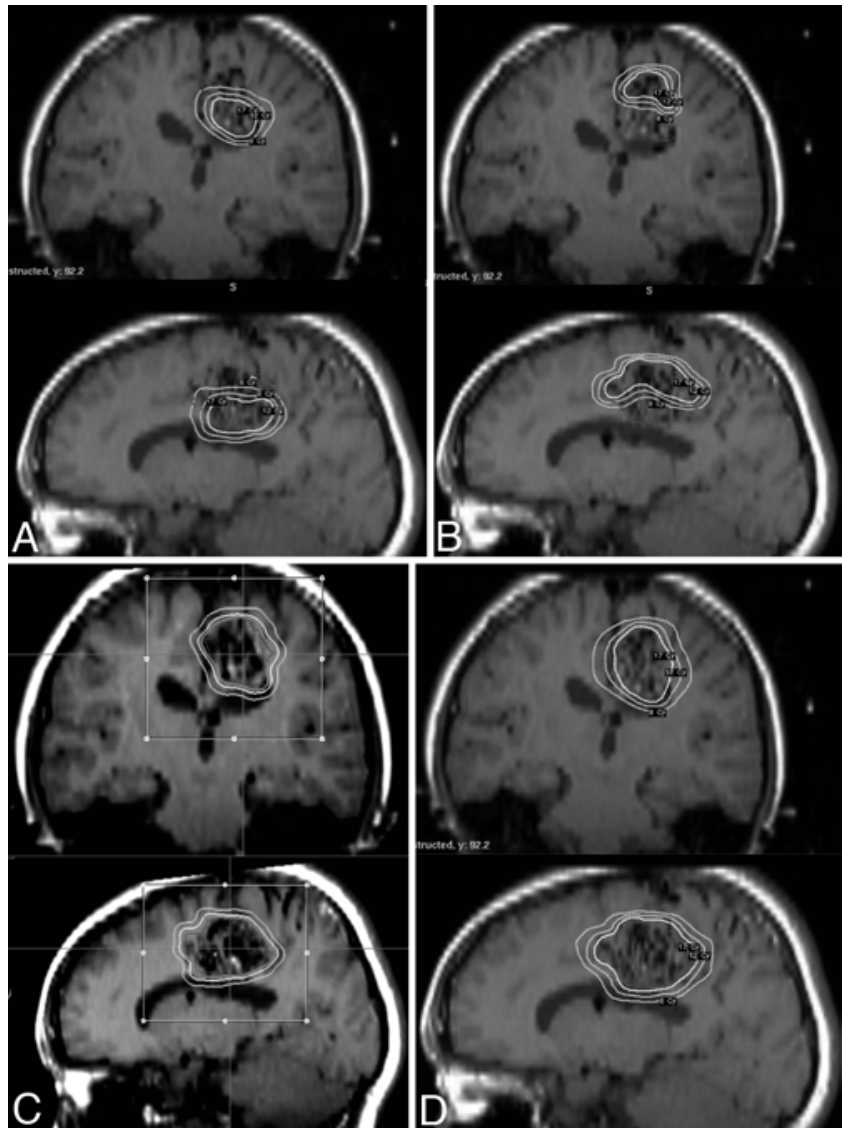

FIG. 1. Representative MR images from a volume-staged SRStreated case in which the Gamma Knife Perfexion was used. A: Isodose distributions are marked for the first stage of treatment. B: Isodose distributions are marked for the second stage of treatment. C: An example of hypofractionated CyberKnife treatment by biologically matching the 12-Gy isodose volumes. D: An example of the hypofractionated Gamma Knife Extend treatment plan, which is identical to the arithmetic sum of the dose distributions shown in panels $A$ and $B$. The three isodose lines shown surrounding the target contours indicate $17 \mathrm{~Gy}, 12 \mathrm{~Gy}$, and 8 Gy moving outward from the center for each panel.

fractions and 24.0 Gy in 4 fractions) are in fact consistently lower than the current hypofractionated schemes reported in the literature. ${ }^{22}$ For example, Xiao et al. ${ }^{24}$ treated 20 large AVMs with 25-30 Gy in 5-6 Gy fractions and found an improvement in efficacy when using $30 \mathrm{~Gy}$. Lindvall et al..$^{15}$ examined 66 patients treated with $30-35$ Gy in 5 fractions and found a higher rate of obliteration in patients treated with 35 Gy than in those treated with 30 Gy $(88 \%$ vs $78 \%)$; however, the authors noted that all patients who experienced symptomatic necrosis belonged to the group treated with $35 \mathrm{~Gy}$. Based on our calculations, 27 Gy may be the appropriate dose to strike a balance between efficacy and toxicity. However, more clinical data with hypofractionated treatments of large AVMs are required. These model calculations need to be further validated before general conclusions can be made about dose efficacy and the potential superiority of hypofractionated treatments over volume-staged treatments.

Our choice of the 12-Gy isodose volume as a dose sur- 
TABLE 1: Summary of treatment planning characteristics in volume-staged Gamma Knife-treated pediatric cases and corresponding hypofractionated full-target CyberKnife treatment plans*

\begin{tabular}{|c|c|c|c|c|c|c|c|c|}
\hline $\begin{array}{l}\text { Target No. \& } \\
\text { Location }\end{array}$ & $\mathrm{V}_{\mathrm{T}}\left(\mathrm{cm}^{3}\right)$ & $\begin{array}{c}\text { Target } \\
\text { Coverage }\end{array}$ & $I \mathrm{SV}_{p}(\mathrm{GK})$ & $\mathrm{ISV}_{\mathrm{p}}(\mathrm{CK})$ & $\mathrm{Cl}(\mathrm{GK})$ & $\mathrm{Cl}(\mathrm{CK})$ & $\begin{array}{c}\text { Hypo SRT 12-Gy } \\
\operatorname{Vol}\left(\mathrm{cm}^{3}\right)\end{array}$ & $\begin{array}{c}\text { Vol-Staged } \\
\text { 12-Gy Vol }\left(\mathrm{cm}^{3}\right)\end{array}$ \\
\hline 1, LPL & 11.3 & $95 \%$ & $50 \%$ & $71 \%$ & 1.36 & 1.43 & $33.4(2.94)$ & $28.2(1.33)$ \\
\hline 2, RFL & 12.1 & $96 \%$ & $50 \%$ & $61 \%$ & 1.45 & 1.41 & $39.2(0.14)$ & $29.7(0.02)$ \\
\hline 3, RFL & 8.0 & $98 \%$ & $50 \%$ & $64 \%$ & 1.34 & 1.28 & $18.1(0.29)$ & $12.6(0.29)$ \\
\hline 4, LTL & 10.0 & $95 \%$ & $50 \%$ & $68 \%$ & 1.24 & 1.25 & $19.8(0.00)$ & $17.5(0.00)$ \\
\hline 5, LOL & 11.8 & $95 \%$ & $50 \%$ & $65 \%$ & 1.41 & 1.40 & $30.0(0.00)$ & $25.9(0.00)$ \\
\hline 6, LFL & 15.0 & $97 \%$ & $50 \%$ & $71 \%$ & 1.33 & 1.25 & $31.5(0.57)$ & $21.3(0.57)$ \\
\hline 7, RFL & 11.2 & $96 \%$ & $50 \%$ & $67 \%$ & 1.46 & 1.32 & $27.9(0.50)$ & $22.8(0.21)$ \\
\hline
\end{tabular}

rogate for normal brain tissue toxicity is well supported by the literature. In our previous studies we demonstrated similar physical peripheral isodose volume characteristics for a solitary target regardless of the isodose volume chosen (for example, 8-Gy, 10-Gy, or 12-Gy isodose volume) or the SRS treatment technology (Gamma Knife, CyberKnife or LINAC-based systems ${ }^{17}$ ). Therefore, using a single peripheral isodose volume (such as the 12-Gy volume in our study) is sufficient as a surrogate for the overall 3-dimensional peripheral dose distribution inside normal brain tissue surrounding a radiosurgical target and, therefore, is not considered a limitation of the study.

In addition, the empirical parameter of $\alpha / \beta=3 \mathrm{~Gy}$ was adopted for the normal brain in our study. Such a value is well supported in the literature, ${ }^{11}$ and small variations in the $\alpha / \beta$ value, such as $10 \%$, were found to introduce negligible changes in the curve shown in Fig. 2. Nonetheless, the application of the linear quadratic model for hypofractionated treatments has been a subject of debate, despite the fact that no other model has yet to be shown and accepted as superior. ${ }^{2}$

One potential limitation of this study is the inabil-

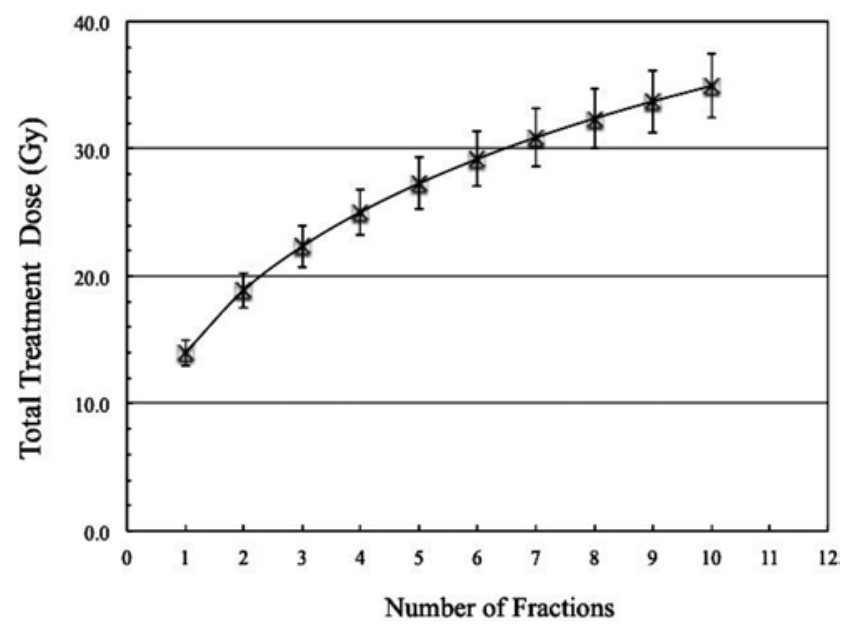

Fig. 2. Plot of total prescribed dose versus total fraction number in the pediatric cases, as derived according to the linear quadratic model in which $\alpha / \beta=3$ Gy was set for the normal brain. ity to account for the relatively long time period (6-9 months) between staged Gamma Knife treatments within the standard linear quadratic model. Our calculations have assumed that the normal brain sparing provided by such an extended time is identical to that provided by daily hypofractionated SRT delivery, but this is unlikely to be accurate. If, on the other hand for example, an extended time period was found to be more beneficial for sparing normal brain compared with daily-administered SRT delivery as expected, then the general conclusion of this study regarding the benefits of staged treatments would be further strengthened.

\section{Conclusions}

The capability of hypofractionated SRTs for large AVMs to spare normal brain is equivalent to that of volume-staged SRS. However, the hypofractionated SRT dose necessary for this was found to be significantly lower than doses reported in current clinical practices. It is only with future clinical comparisons that we will know whether more patients have been subjected to higher rates of radiation necrosis than currently observed using volume-staged treatments.

\section{Disclosure}

The authors acknowledge technical and equipment support provided by Elekta and Accuray for the study. Dr. McDermott shares a copyright with the University of California, San Francisco, for a medical device. Dr. Larson is a stockholder and member of the board of directors for American Shared Hospital Services.

Author contributions to the study and manuscript preparation include the following. Conception and design: Ma, Sneed, McDermott, Larson. Acquisition of data: Ma, Fogh, Gupta, Nakamura, Barani, Sneed, McDermott. Analysis and interpretation of data: Ma, Fogh, Gupta, Sahgal, McDermott, Larson. Drafting the article: Ma, Fogh, Sahgal, Larson. Critically revising the article: all authors. Reviewed submitted version of manuscript: all authors. Approved the final version of the manuscript on behalf of all authors: Ma. Statistical analysis: Ma, Sahgal. Administrative/technical/material support: Ma, Gupta, Nakamura, Barani, Sneed, McDermott, Larson. Study supervision: Ma, Sneed, McDermott. 


\section{Hypofractionation versus volume staging for treating large AVMs}

\section{References}

1. Adler JR Jr, Chang SD, Murphy MJ, Doty J, Geis P, Hancock SL: The Cyberknife: a frameless robotic system for radiosurgery. Stereotact Funct Neurosurg 69:124-128, 1997

2. Brenner DJ: The linear-quadratic model is an appropriate methodology for determining isoeffective doses at large doses per fraction. Semin Radiat Oncol 18:234-239, 2008

3. Cernica G, de Boer SF, Diaz A, Fenstermaker RA, Podgorsak MB: Dosimetric accuracy of a staged radiosurgery treatment. Phys Med Biol 50:1991-2002, 2005

4. Chang TC, Shirato H, Aoyama H, Ushikoshi S, Kato N, Kuroda S, et al: Stereotactic irradiation for intracranial arteriovenous malformation using stereotactic radiosurgery or hypofractionated stereotactic radiotherapy. Int J Radiat Oncol Biol Phys 60:861-870, 2004

5. Colombo F, Cavedon C, Casentini L, Francescon P, Causin F, Pinna V: Early results of CyberKnife radiosurgery for arteriovenous malformations. Clinical article J Neurosurg 111: 807-819, 2009

6. Flickinger JC, Kondziolka D, Pollock BE, Maitz AH, Lunsford LD: Complications from arteriovenous malformation radiosurgery: multivariate analysis and risk modeling. Int J Radiat Oncol Biol Phys 38:485-490, 1997

7. Flickinger JC, Lunsford LD, Kondziolka D, Maitz AH, Epstein AH, Simons SR, et al: Radiosurgery and brain tolerance: an analysis of neurodiagnostic imaging changes after gamma knife radiosurgery for arteriovenous malformations. Int J Radiat Oncol Biol Phys 23:19-26, 1992

8. Karlsson B, Lax I, Soderman M: Factors influencing the risk for complications following Gamma Knife radiosurgery of cerebral arteriovenous malformations. Radiother Oncol 43: 275-280, 1997

9. Karlsson B, Lindqvist M, Blomgren H, Wan-Yeo G, Söderman M, Lax I, et al: Long-term results after fractionated radiation therapy for large brain arteriovenous malformations. Neurosurgery 57:42-49, 2005

10. Kihlström L, Guo WY, Karlsson B, Lindquist C, Lindqvist $\mathrm{M}$ : Magnetic resonance imaging of obliterated arteriovenous malformations up to 23 years after radiosurgery. J Neurosurg 86:589-593, 1997

11. Lawrence YR, Li XA, el Naqa I, Hahn CA, Marks LB, Merchant TE, et al: Radiation dose-volume effects in the brain. Int J Radiat Oncol Biol Phys 76 (3 Suppl):S20-S27, 2010

12. Lax I, Karlsson B: Prediction of complications in gamma knife radiosurgery of arteriovenous malformation. Acta Oncol 35:49-55, 1996

13. Lindquist C, Guo WY, Karlsson B, Steiner L: Radiosurgery for venous angiomas. J Neurosurg 78:531-536, 1993

14. Lindquist C, Paddick I: The Leksell Gamma Knife Perfexion and comparisons with its predecessors. Neurosurgery 61 (3 Suppl):130-141, 2007

15. Lindvall $\mathrm{P}$, Bergström $\mathrm{P}$, Blomquist $\mathrm{M}$, Bergenheim AT: Radiation schedules in relation to obliteration and complications in hypofractionated conformal stereotactic radiotherapy of arteriovenous malformations. Stereotact Funct Neurosurg 88:24-28, 2010

16. Lindvall P, Bergström P, Löfroth PO, Henriksson R, Bergen- heim AT: Hypofractionated conformal stereotactic radiotherapy alone or in combination with whole-brain radiotherapy in patients with cerebral metastases. Int J Radiat Oncol Biol Phys 61:1460-1466, 2005

17. Ma L, Sahgal A, Descovich M, Cho YB, Chuang C, Huang $\mathrm{K}$, et al: Equivalence in dose fall-off for isocentric and nonisocentric intracranial treatment modalities and its impact on dose fractionation schemes. Int J Radiat Oncol Biol Phys 76:943-948, 2010

18. Petti PL, Coleman J, McDermott M, Smith V, Larson DA: Anatomic landmarks versus fiducials for volume-staged gamma knife radiosurgery for large arteriovenous malformations. Int J Radiat Oncol Biol Phys 67:1578-1585, 2007

19. Pollock BE, Kline RW, Stafford SL, Foote RL, Schomberg PJ: The rationale and technique of staged-volume arteriovenous malformation radiosurgery. Int J Radiat Oncol Biol Phys 48:817-824, 2000

20. Sinclair J, Chang SD, Gibbs IC, Adler JR Jr: Multisession CyberKnife radiosurgery for intramedullary spinal cord arteriovenous malformations. Neurosurgery 58:1081-1089, 2006

21. Smyth MD, Sneed PK, Ciricillo SF, Edwards MS, Wara WM, Larson DA, et al: Stereotactic radiosurgery for pediatric intracranial arteriovenous malformations: the University of California at San Francisco experience. J Neurosurg 97:4855,2002

22. Veznedaroglu E, Andrews DW, Benitez RP, Downes MB, Werner-Wasik M, Rosenstock J, et al: Fractionated stereotactic radiotherapy for the treatment of large arteriovenous malformations with or without previous partial embolization. Neurosurgery 55:519-531, 2004

23. Wowra B, Muacevic A, Tonn JC, Schoenberg SO, Reiser M, Herrmann KA: Obliteration dynamics in cerebral arteriovenous malformations after cyberknife radiosurgery: quantification with sequential nidus volumetry and 3-tesla 3-dimensional time-of-flight magnetic resonance angiography. Neurosurgery 64 (2 Suppl):A102-A109, 2009

24. Xiao F, Gorgulho AA, Lin CS, Chen CH, Agazaryan N, Viñuela $\mathrm{F}$, et al: Treatment of giant cerebral arteriovenous malformation: hypofractionated stereotactic radiation as the first stage. Neurosurgery 67:1253-1259, 2010

25. Zabel-du Bois A, Milker-Zabel S, Huber P, Schlegel W, Debus J: Linac-based radiosurgery or hypofractionated stereotactic radiotherapy in the treatment of large cerebral arteriovenous malformations. Int J Radiat Oncol Biol Phys 64:1049-1054, 2006

Manuscript submitted May 16, 2012.

Accepted July 10, 2012.

This work was presented in part at the 16th International Meeting of the Leksell Gamma Knife Society, Sydney, Australia.

Please include this information when citing this paper: DOI: 10.3171/2012.7.GKS121023.

Address correspondence to: Lijun Ma, Ph.D., University of California, San Francisco, 505 Parnassus Avenue, Room L-08, San Francisco, California 94143.email: lijunma@radonc.ucsf.edu. 